\title{
Picrasidine I from Picrasma Quassioides Suppresses Osteoclastogenesis via Inhibition of RANKL Induced Signaling Pathways and Attenuation of ROS Production
}

\author{
Lingbo Kong Biao Wang Xiaobin Yang Hua Guo Ke Zhang Ziqi Zhu \\ Jijun Liu Dingjun Hao
}

Hong-Hui Hospital, Xi'an Jiaotong University College of Medicine, Xi'an Shaanxi, China

\section{Key Words}

Picrasma Quassioides • Reactive oxygen species (ROS) • Osteoclastogenesis

\begin{abstract}
Background/Aims: Osteoporosis is a metabolic bone disorder that tortures about millions of people worldwide. Recent study demonstrated agents derived from picrasma quassioides is a promising drug for targets multiple signaling pathways. However its potential in treatment of bone loss has not been fully understood. Methods: The bone marrow macrophages (BMMs) were cultured and induced with M-CSF and RANKL followed by picrasidine I (PI) treatment. Then the effects of PI on osteoclast formation were evaluated by counting tartrate-resistant acid phosphatase (TRAP)-positive multinucleated cells. Moreover, effects of PI on bone resorption activity of mature osteoclast were studied through bone resorption pit counting and actin ring structure analysis. Further, the involved potential signaling pathways cross-talking were investigated by performed Western blotting and quantitative real-time PCR examination. Results: Results demonstrated PI strongly inhibited RANKL induced osteoclast formation from its precursors. Mechanistically, the inhibitory effect of PI on osteoclast differentiation was due to the suppression of osteoclastogenic transcription factors, c-Fos and NFATc1. Moreover, PI markedly blocked the RANKL-induced osteoclastogenesis by attenuating MAPKs and NF-KB signaling pathways. In addition, PI decreased the ROS generation in osteoclast and osteoblast. Conclusion: Taken together our data demonstrate that PI has antiosteoclastogenic effect by inhibiting inflammation induced activation of MAPKs, NF-KB and ROS generation followed by suppressing the gene expression of c-Fos and NFATc1 in osteoclast precursors.




\section{Cellular Physiology Cell Physiol Biochem 2017;43:1425-1435 \begin{tabular}{l|l|l} 
and Biochemistry Published online: October 10, 2017 & $\begin{array}{l}\text { (c) } 2017 \text { The Author(s). Published by S. Karger AG, Basel } \\
\text { www.karger.com/cpb }\end{array}$ \\
\hline
\end{tabular} \\ Kong et al.: Agent from Picrasma Quassioides Suppress Osteoclastogenesis}

\section{Introduction}

Osteoclasts (OCs) are multinucleated bone-resorbing cells that are unique in their ability to degrade mineralized matrices, such as bone and calcified cartilage [1]. Although many systemic hormones and local cytokines participate in regulating osteoclast differentiation (osteoclastogenesis), the receptor activator of NF- $\kappa B$ (RANK) ligand (RANKL) is the most critical molecular for osteoclastogenesis in cooperation with macrophage colony-stimulating factor (M-CSF) in the interaction between stromal cells and cells of the OCs lineage $[2$, 3]. Binding of RANKL to cell surface receptor RANK sequentially activate downstream signaling molecules including: the nuclear factor- $\kappa \mathrm{B}(\mathrm{NF}-\kappa \mathrm{B})$ and mitogen activated protein kinases (MAPKs) [4]. The MAPK family includes p38, signal-regulated kinase (ERK) and c-jun-Nterminal kinase (JNK) [5]. Activation of MAPKs signaling further up regulates the expression of c-Fos, which in turn, activation of nuclear factor of activated T cells c1 (NFATc1) [6-8]. Moreover, previous study reported that small amounts of reactive oxygen species ROS acts as secondary messengers and activates JNK and p38 signalling pathways [9]. RANKL stimulation in OCs precursors increases the ROS generation mediated through TRAF6, Rac1, and NADPH (nicotinamide adenine dinucleotide phosphate) oxidase 1 (Nox1) and further enhances the osteoclastogenesis [10].

Recently, compound screening for plant-derived natural products exhibit minimum side effects and are available in cost effective manner, therefore a number of research groups have carried out screening for osteoclastogenesis, which has gained considerable attention [11]. Picrasidine I (PI) is a primary active constituent of traditional Chinese medicine from picrasma quassioides [12]. Previously, it has been reported that agent derived from picrasma quassioides has anti-inflammatory properties and exerts beneficial effects in the cerebral [13]. Moreover, most recent studies have demonstrated picrasidine $\mathrm{N}$ and picrasidine G target multiple signaling pathways, such as: PARP-1and EGFR/STAT3 signaling pathway $[14,15]$. However, the effects of PI on ROS which cross talking in the process of RANKL-stimulated osteoclastogenesis and the influence of PI on osteogenic have not been investigated. Herein, we aimed to deeply explore the effects of PI on signaling pathway involved in osteoclastogenesis and its underlying molecular mechanisms in vitro.

\section{Materials and Methods}

Ethics approval and consent to participate

All animal care and experimental procedures were approved by Animal Care Committee of HongHui Hospital, Xi'an Jiaotong University College of Medicine (Animal Ethics Approval \#1002016019) and conducted strictly followed by "the institutional guidelines for the care and use of laboratory animals at the Jiaotong University College of Medicine “.

\section{Reagents and antibodies}

Compound (Picrasidine I, Fig. 1, purity $\geq 95 \%$, CAS No. 100234-59-1, Catalog No. CFN99019, molecular weight 240.3) was purchased from ChemFaces co. (Wuhan, China). The primary stock solution of PI was prepared by dissolving an appropriate amount in of PI in $5 \mathrm{ml}$ dichloromethane. Then, the stock solutions were diluted with methanol to obtain a series of working standard solutions at the concentrations of 2, 10 and $20 \mu \mathrm{M}$. All stock solutions and working standard solutions were stored at $-20^{\circ} \mathrm{C}$ until use. Recombinant human M-CSF and RANKL were obtained from PeproTech EC, Ltd. (London, U.K.). The XTT assay kit was obtained from Roche (Indianapolis, IN, USA). Antibodies for c-Fos and NFATc1 were purchased from Santa Cruz Biotechnology (Santa Cruz, CA, USA). Specific primary antibodies against phospho-p38, p38, phospho-

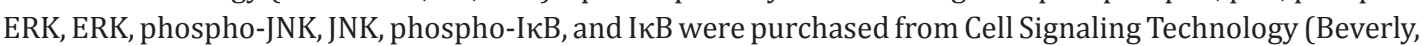
MA, USA); and specific inhibitors, U-0126, SB-203580 and SP-600125 were purchased from Calbiochem (CA, USA). $\beta$-actin antibody was purchased from Sigma-Aldrich, Inc. (St. Louis, MO, USA).

Cell lines and in vitro osteoclastogenesis assay

Three cell lines were used in the present study, RAW 264.7 and MC-3T3-E1 cells were obtained from American Type Culture Collection (Manassas, VA). Besides, BMMs were prepared as described previously 


\section{Cellular Physiology Cell Physiol Biochem 2017;43:1425-1435 \begin{tabular}{l|l|l} 
DOI: 10.1159/000481874 & $\begin{array}{l}\text { O 2017 The Author(s). Published by S. Karger AG, Basel } \\
\text { www.karger.com/cpb }\end{array}$
\end{tabular}}

[16]. Briefly, RAW264.7 cells were cultured in DMEM, BMMs and MC-3T3-E1 cells were cultured in $\alpha$-MEM. Medias were supplemented with $10 \%$ fetal bovine serum, penicillin and streptomycin. Then BMMs were cultured in the presence of M-CSF $(20 \mathrm{ng} / \mathrm{ml})$ and RANKL $(40 \mathrm{ng} / \mathrm{ml})$ for 4 days in the presence or absence of P I $(0,2,10$ or $20 \mu \mathrm{M})$. The multinucleated cells (more than 3 nuclei) were counted using phage contrast microscope at $100 \times$ magnification. Cell images were obtained using a digital camera attached to a Nikon ECLIPSE TE2000-S microscope (Nikon, Tokyo, Japan).

Cell viability assays, cytotoxicity assay and cell apoptosis assay

Cytotoxicity of PI on BMMs were performed as our previously described $[17,18]$. Briefly, BMMs were seeded at a density of $3.5 \times 10^{3}$ cells per well in 96-well plates with various concentrations of PI and incubated for $3 \mathrm{~d}$ in the presence of RANKL. XTT solution was added to each well and incubated for $4 \mathrm{~h}$. The plate was read at $450 \mathrm{~nm}$. Meanwhile, after the PI treatments, the released LDH collected by culture supernatants. Subsequently, measured by an enzymatic assay, which is based on a color change at $490 \mathrm{~nm}$ according to manufacturer's instruction. Cell apoptosis were assessed by TUNEL assay kits according to manufacturer's instruction, and number of apoptotic cells were count by fluorescent microscopy (LSM700, Carl Zeiss, Oberkochen, Germany). Relative fluorescence was determined using a multi-detection microplate reader ( $485 \mathrm{~nm}$ excitation and $535 \mathrm{~nm}$ emission).

\section{Bone absorption assay and Fibrous actin ring immunofluorescence}

Mature OCs function evaluated by bone absorption assay [19] and F-actin ring immunofluorescence as previously described respectively [17]. Briefly, cells were treated with $50 \mathrm{ng} / \mathrm{ml}$ RANKL on 6-well plates pre-coated with collagen for 4 days. Here, $2.5 \mathrm{mg} / \mathrm{ml}$ collagenase in dissociation buffer was used to collect mature osteoclasts, which were seeded onto a Corning OsteoAssay Surface (Newyork, US) in a multiple-well plate. Mature OCs were then treated with various concentrations of PI or vehicle in the presence of RANKL $(40 \mathrm{ng} / \mathrm{ml})$ for 3 days. Five percent sodium hypochlorite was used to wash the disc for $5 \mathrm{~min}$. The resorption area was quantified using image analysis (Olympus Soft Imaging Solutions, Munster, Germany). One day later, the cells were fixed in $3.7 \%$ formalin for $20 \mathrm{~min}$, permeabilized with $0.1 \%$ TritonX-100 for $15 \mathrm{~min}$, incubated with $0.25 \%$ bovine serum albumin for $30 \mathrm{~min}$, the cells were stained with DAPI for $2 \mathrm{~min}$ and then photographed using fluorescence microscope (Nikon) at $\times 200$ magnification.

\section{Quantitative real-time reverse transcription polymerase chain reaction (RT-PCR)}

Total RNA was isolated from cell culture via QIAzol reagent (QIAGEN, Valencia, CA, U.S.A.) according to the manufacturer's instructions RNA $(1 \mu \mathrm{g})$ was reverse transcribed using oligo dT primers $(10 \mu \mathrm{g})$ and dNTPs $(10 \mathrm{mM})$. The mixture was incubated at $65^{\circ} \mathrm{C}$ for $5 \mathrm{~min}$, and cDNA was produced by incubating at $42^{\circ} \mathrm{C}$ for 50 min with first strand buffer ( $50 \mathrm{mM}$ Tris- $\mathrm{HCl}, \mathrm{pH} 8.3,75 \mathrm{mM} \mathrm{KCl}, 3 \mathrm{mM} \mathrm{MgCl}$ ), $100 \mathrm{mM}$ DTT, RNase inhibitor, and Superscript II reverse transcriptase (Invitrogen). The specific primers used to amplify genes were presented in Table.1. The mouse GAPDH gene was used as internal control.

\section{Western blotting}

Cells treated for the indicated days with various concentrations of PI were washed with cold PBS and lysed with radio immune precipitation assay (RIPA) buffer plus phenylmethylsulfonyl fluoride (PMSF). Cell lysates were centrifuged at $12,000 \mathrm{rpm}$ for $10 \mathrm{~min}$, and supernatants were collected as samples. Protein (30 $\mathrm{mg}$ ) was separated on $10 \%$ SD PAGE and transferred to polyvinylidene fluoride (PVDF) membranes. The membranes were blocked with 5\% skim milk in TBST containing $0.05 \%$ Tween-20 and probed successively with anti NFATc1, c-Fos antibodies overnight at $4{ }^{\circ} \mathrm{C}$. Horseradish peroxidase-conjugated rabbit anti-

Table 1. Primer sequences used for real-time RT-PCR analysis

\begin{tabular}{lcc}
\hline Gene Name & Primer Sequence (5'-3') Forward & Primer Sequence (5'-3') Reverse \\
\hline c-Fos & $5^{\prime}$-CTGGTGCAGCCCACTCTGGTC- $3^{\prime}$ & $5^{\prime}$-CTTTCAGCAGATTGGCAATCTC-3' \\
NFATc1 & 5'-CTCGAAAGACAGCACTGGAGCAT-3' $^{\prime}$ & $5^{\prime}$-CGGCTGCCTTCCGTCTCATAG-3' \\
GAPDH & 5'-TCA AGA AGG TGG TGA AGC AG-3' $^{\prime}$ & 5'-AGT GGG AGT TGC TGT TGA AGT-3' $^{\prime}$ \\
\hline
\end{tabular}




\section{Cellular Physiology Cell Physiol Biochem 2017;43:1425-1435 \begin{tabular}{l|l|l} 
DOI: 10.1159/000481874 & Ond Biochemistry Published online: October 10, 2017 & $\begin{array}{l}\text { 2 } 2017 \text { The Author(s). Published by S. Karger AG, Basel } \\
\text { www.karger.com/cpb }\end{array}$
\end{tabular} \\ Kong et al.: Agent from Picrasma Quassioides Suppress Osteoclastogenesis}

mouse IgG antibodies were used as the secondary antibodies for $1 \mathrm{~h}$ at room temperature. The signals were detected by exposure in an Odyssey infrared imaging system (LI-COR).

\section{Immunofluorescence analysis}

Appropriately treated RAW 264.7 cells grown on glass coverslips were fixed with $4 \%$ paraformaldehyde, air-dried, and stored at $-20^{\circ} \mathrm{C}$ until use. Cells were washed with PBS, and blocked in $10 \%$ non-immune goat serum for $1 \mathrm{~h}$ at room temperature. Then cells were incubated with mouse monoclonal antibody against NF$\mathrm{\kappa B} \mathrm{p} 65$ or IgG isotype control antibody at a final dilution of $10 \mathrm{ug} / \mathrm{ml}$ at $4^{\circ} \mathrm{C}$ overnight washed twice with tris-buffered saline followed by incubation with TRITC-conjugated goat anti-mouse IgG (1:100) or FITCconjugated rabbit anti-goat IgG (1:100) for $1 \mathrm{~h}$ at room temperature. Nuclei were stained with DAPI, and coverslips were mounted on a microscope slide with embedding medium.

\section{ROS products measurement}

Intracellular ROS generation was measured as previously described [20]. The RAW264.7 and MC-3T3-E1 cells were seeded into a 96-black well plate for $24 \mathrm{~h}$. After pretreatment with the indicated concentrations of P I ( 5 and $20 \mu \mathrm{M}$ ) for $6 \mathrm{~h}$, cells were induced by RANKL ( $40 \mathrm{ng} / \mathrm{ml}$ ) for $10 \mathrm{~min}$ and $\mathrm{H}_{2} \mathrm{O}_{2}$ $(250 \mathrm{uM})$ for $2 \mathrm{~h}$ respectively. After treatment, cells were incubated with $10 \mathrm{uM}$ of cell permeant reagent 2',7'- dichlorofluorescein diacetate (DCF-DA) at $37^{\circ} \mathrm{C}$ for $15 \mathrm{~min}$. The ROS mediated oxidation of DCF-DA to the fluorescent compound 2',7'-dichlorofluorescein (DCF) was monitored by fluorescence microscopy and Multimode Plate Reader (Spectra Max M4, Molecular devices, USA) at excitation (498 nm) and emission $(530 \mathrm{~nm})$.

\section{Statistical Analysis}

Differences among groups were statistically analyzed using one-way ANOVA; Bonferroni's multiple comparison test was used as post-test analyses. $\mathrm{P}<0.05$ was considered to be significant. Data are reported as mean \pm SEM for each group.

\section{Results}

PI Inhibits RANKL-Induced OCs Differentiation without significant toxic effects

To assess the effect of PI on OCs differentiation, we induced osteoclastogenesis in BMMs in the absence or presence of PI at concentrations as indicated. After induced by M-CSF and RANKL in the culture, OCs started to form as shown by the presence of TRAP-positive multinucleated cells. However, administration with PI strongly inhibited OC formation with the respect of size and number respectively (Fig. 1B; Fig. 1C). Because the suppressive effect on osteoclast formation might have been due to cytotoxicity of this compound, we used the XTT, LDH and TUNEL assay on BMMs cultured with various doses of PI for $3 \mathrm{~d}$. At the concentrations used in this study, PI did not exert cytotoxic effects (Fig. 1E; Fig. 1F).

\section{PI decreases OCs activity and function in vitro}

Pit formation assays were performed to assess OCs activity. Mature OCs were seeded on an osteo assay surface in the presence of RANKL and treated with different concentrations of PI. As results shown that PI inhibits bone resorption activity of OCs (Fig. 2A: a-d; Fig. 2B). Moreover, actin ring structure is a characteristic cytoskeletal feature of functional OCs [21]. Therefore, we examined whether PI affects actin ring structure of mature OCs. In mature OCs on tissue culture plates, F-actin was arranged into a ring-like structure (actin ring) at the cell periphery. Treatment of PI caused both shrinkage of mature OCs and disruption of actin ring structure in a dose-dependent manner (Fig. 2A: e-h; Fig. 2C).

PI inhibits RANKL-induced expression of NFATc1 and c-Fos

c-Fos and NFATc1 are two key transcription factors in OCs differentiation [22]. To clarify the molecular mechanism of inhibitory effects of PI on osteoclastogenesis, the expression levels of c-Fos and NFATc1 were examined via real-time PCR and immunoblotting assay, 


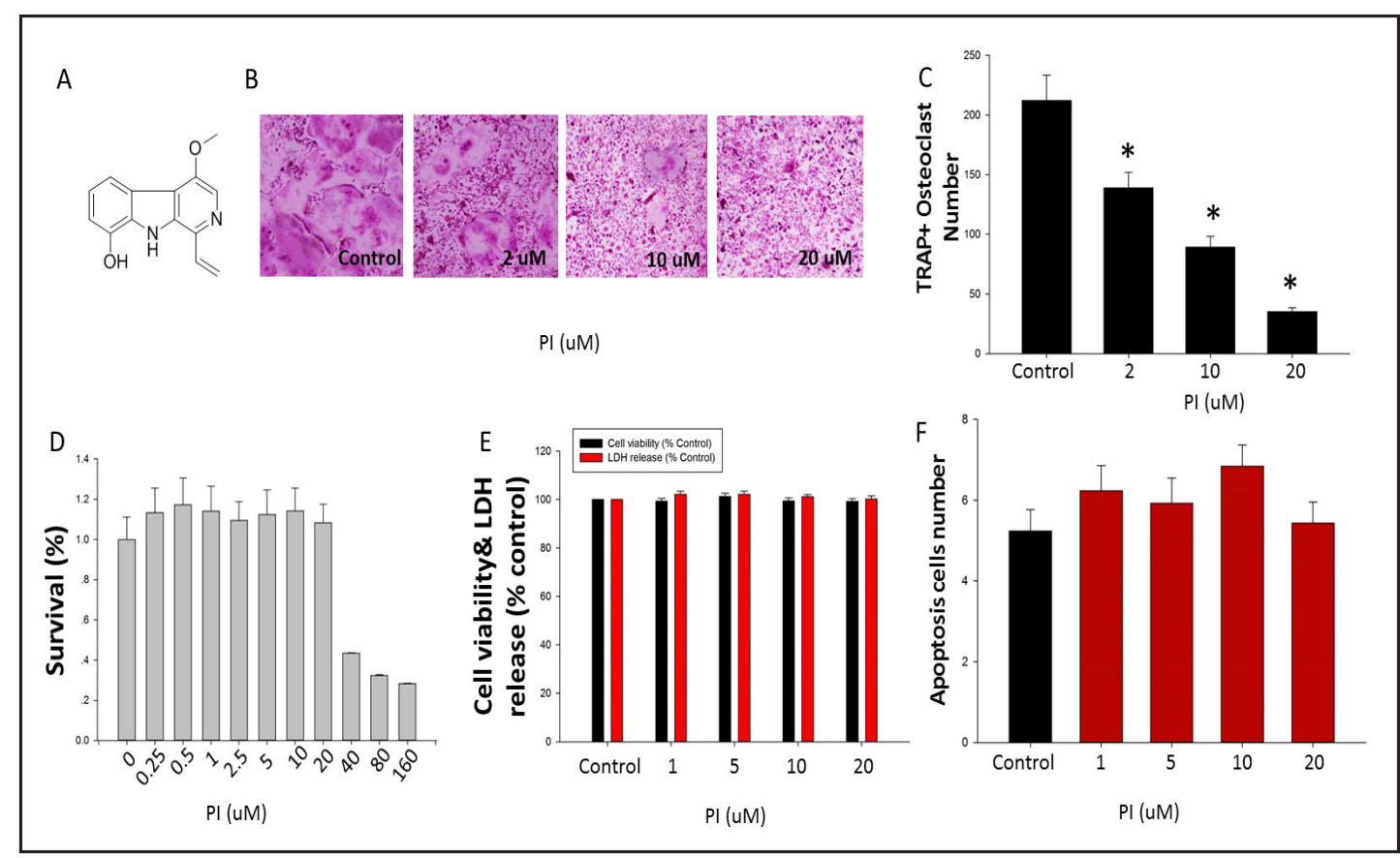

Fig. 1. Effect of PI on RANKL-induced OCs differentiation. (A) Chemical structure of PI. (B) TRAP-positive cells were counted as osteoclasts in each experimental group. (C) Number of TRAP-positive OCs. ${ }^{*}: \mathrm{p}<0.05$ vs. control. (D) Cell survival rate, (E) Relative cell viability and LDH release and (F) apoptosis cell number showed there were no significant differences with PI treatment. Similar results were obtained in at least 3 independent experiments.

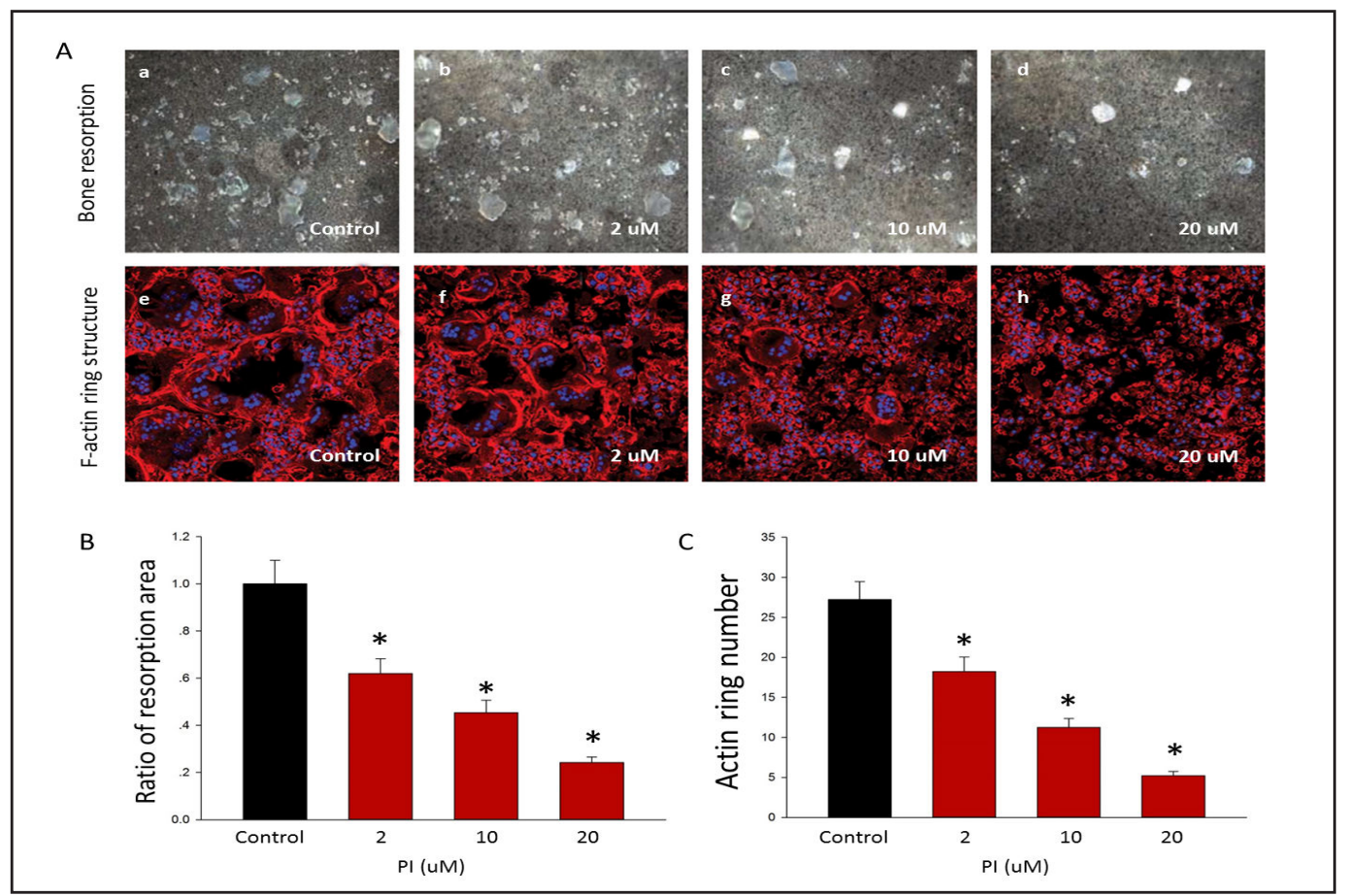

Fig. 2. PI inhibited osteoclast bone resorption and F-actin ring structure. (A) a-d: Images of mature osteoclast erosion pits were inhibited by PI; e-h: Cells were fixed and stained for F-actin (B) The ration of Resorption pit area. (C) Number of actin ring. All experiments were performed at least three times, and the significance was determined as indicated in methods ( $:$ p $<0.05$ vs. control). 


\section{Cellular Physiology and Biochemistry

Fig. 3. Effect of PI on RANKLinduced the mRNA expression of c-Fos and NFATc1. (A) BMMs were pretreated with or without PI for $1 \mathrm{~h}$ and with RANKL (100 $\mathrm{ng} / \mathrm{ml}$ ) for the indicated time. The mRNA expression of c-Fos and NFATc1 genes was analyzed by real time RT-PCR. (B) Western blotting analysis showed PI inhibits the expression of c-Fos and NFATc1 induced by RANKL. The intensities of the protein bands were analyzed and normalized to actin. Similar results were obtained in at least 3 independent experiments.

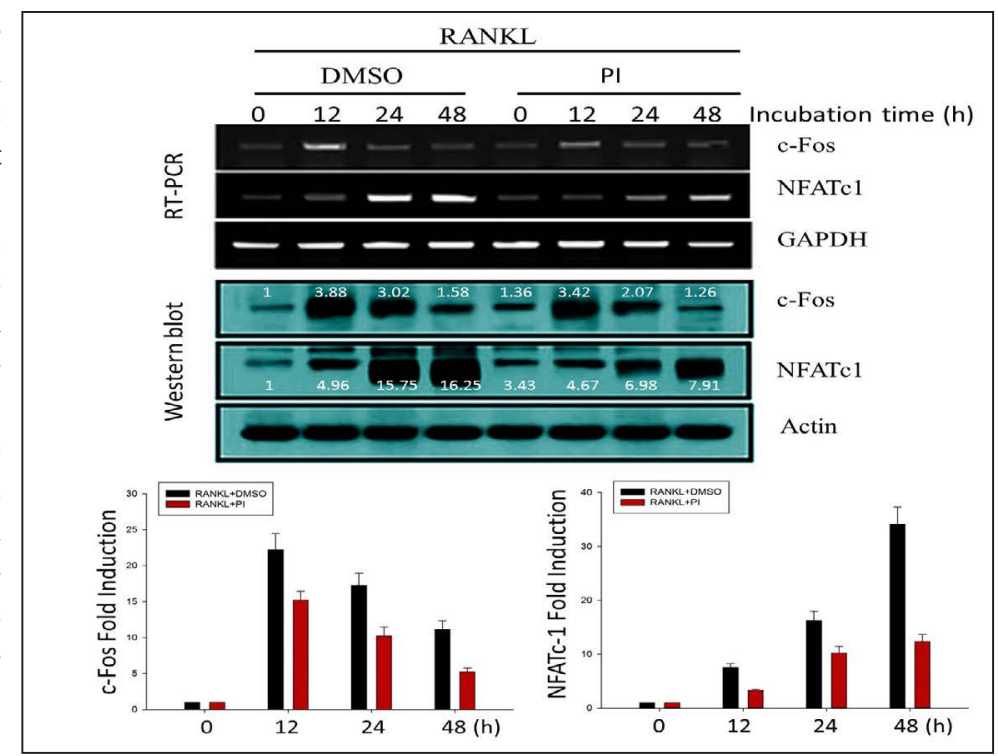

Fig. 4. PI inhibits MAPKs signaling pathways. (A) Western blot analysis for expression and phosphorylation levels of ERK, p38, JNK of BMMs induced by RANKL followed by PI treatment or MAPK inhibitors, SB-203580 (SB), U-0126 (U), SP-600125 (SP). The intensities of protein bands were analyzed and normalized to actin. (B) Rrelative ratios of protein levels (down) are shown. *: p< 0.05 vs. RANKL. Similar results were obtained in at least 3 independent experiments.

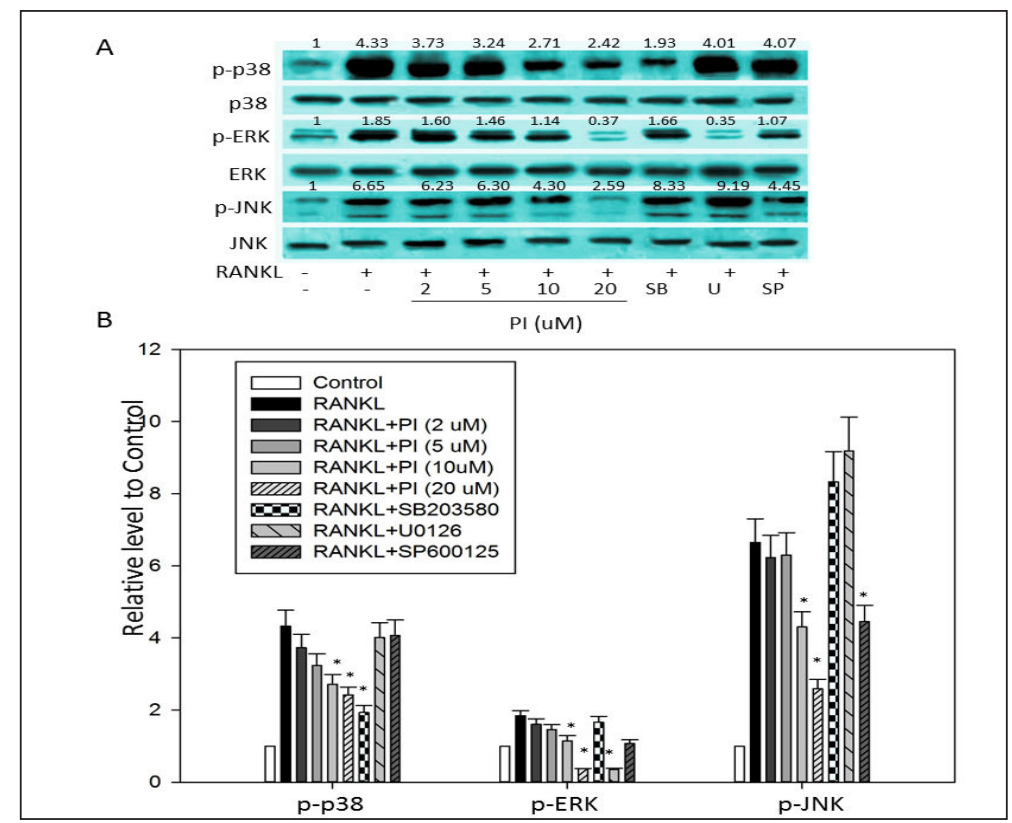

respectively. As shown in Fig. 3, the mRNA levels of c-Fos and NFATc1 were significantly increased by RANKL stimulation. However, with the administration of PI, the mRNA expression of c-Fos and NFATc1markedly decreased. Correspondingly, PI treatment also significantly reduced RANKL-induced expression of c-Fos and NFATc1 at protein levels.

\section{Effects of PI on RANKL-initiated MAPKs activation}

As previously reported, activation of the MAPKs/c-Fos/NFATc1 pathway plays a pivotal role in osteoclastogenesis [23]. To evaluate the effects of PI on MAPKs following the stimulation of RANKL in BMMs, we employed three MAPK specific inhibitors (U-0126, SB203580 and SP-600125) to investigate the role of PI inhibitory effects on phosphorylation of p38, JNK, and ERK, respectively. Our results showed that PI significantly inhibited RANKLinduced phosphorylation of p38, JNK and ERK (Fig. 4). These results indicate that MAPKs/cFos/NFATc1 partly involved in the cross-talking of PI inhibitory effects on RANKL-induced osteoclastogenesis. 
Fig. 5. Effects of PI on RANKLactivated $\mathrm{I} \kappa \mathrm{B} / \mathrm{NF}-\kappa \mathrm{B} \quad$ signaling pathway. (A) Immunofluorescence staining was performed to locate NF- $\kappa$ B p65 (red). Nuclear counterstaining was performed using DAPI (blue). (B) RAW 264.7 cells were pretreated by PI $(0$, $2,10,20 \mu \mathrm{M}$ ) for $2 \mathrm{~h}$, and then treated with $100 \mathrm{ng} / \mathrm{ml}$ RANKL for $30 \mathrm{~min}$. Total, cytoplasmic, or nuclear protein was extracted, and subjected to Western blot analysis using antibodies against phospho-I $\kappa \mathrm{B}$, I $\mathrm{\kappa}$ B, NF- $\kappa$ B p65, or actin. Similar results were obtained in at least 3 independent experiments.

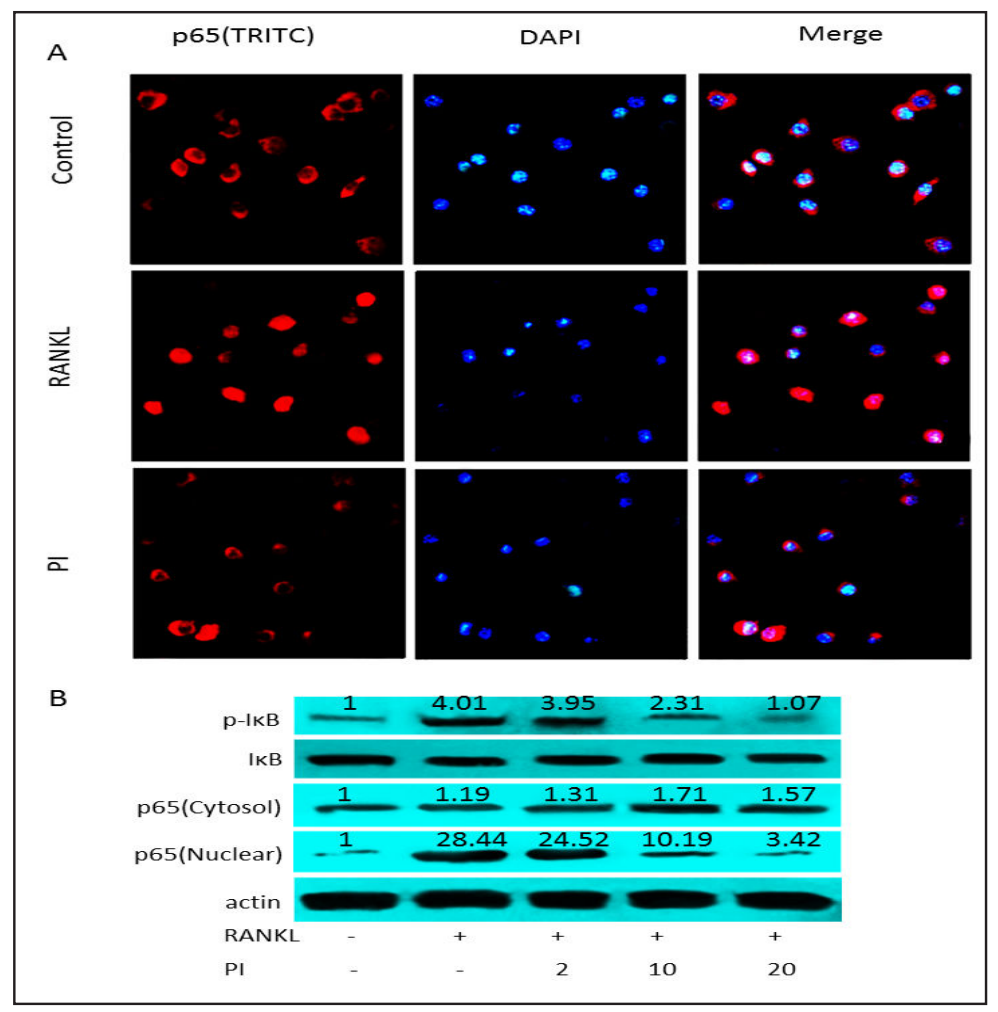

Fig. 6. Effect of PI on ROS production in RAW264.7 cells and MC-3T3-E1 cells. (A) ROS production in both RAW 264.7 and MC-3T3-E1 cells were reversed by PI treatment; (B) Relative DCF fluorescence analysis for MC-3T3-E1 and (C) RAW 264.7 cells respectively. *: p< 0.05 vs. control, \#: $\mathrm{p}<0.05$ vs. RANKL; \#\#: $\mathrm{p}<0.05$ vs. $\mathrm{H}_{2} \mathrm{O}_{2}$. Similar results were obtained in at least 3 independent experiments.

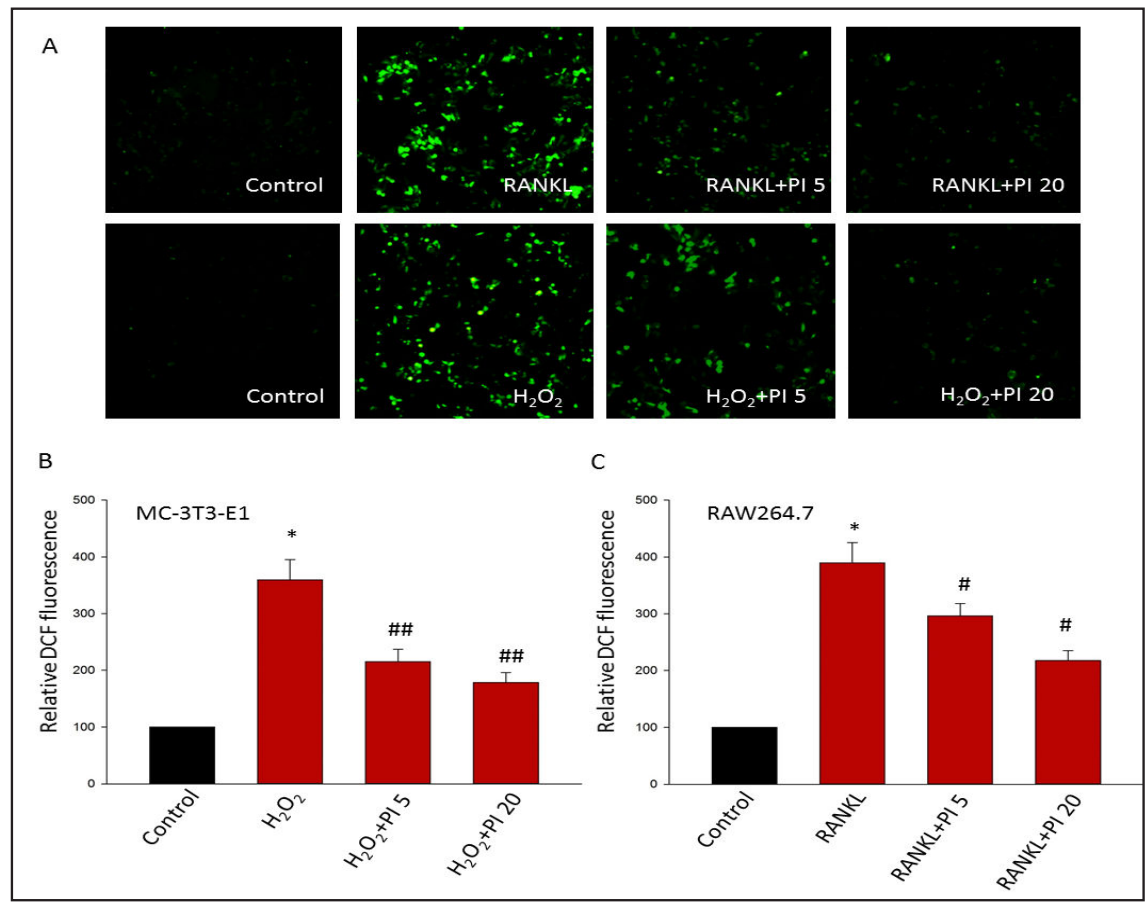

\section{Effects of PI on RANKL-activated I $\kappa B / N F-\kappa B$ signaling pathways}

RANKL-induced NF- $\kappa B$ activation is essential in initiating OCs differentiation [24]. To further explore the mechanisms of PI inhibited NF-kB-mediated osteoclastogenesis, we employed two different methods (Western blotting and immunofluorescence staining). As shown in Fig. 5, in the absence of RANKL, most p65 units were located in the cytoplasm, 


\section{Cellular Physiology Cell Physiol Biochem 2017;43:1425-1435

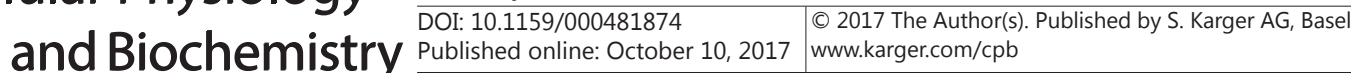 \\ Kong et al.: Agent from Picrasma Quassioides Suppress Osteoclastogenesis}

which was unphosphorylated and inactive. Upon RANKL stimulation, almost all p65 units were located in the nucleus. However, the RANKL induced p65 nuclear translocation was blocked when pretreated with PI. Both two methods indicated that PI could inhibit RANKLmediated activation of the NF- $\mathrm{K}$ B pathway.

\section{PI decreases the release of ROS}

As ROS is a key intracellular mediator for signaling pathways cross talking in osteoclastogenesis, we examined ROS production in RANKL stimulated RAW264.7 and $\mathrm{H}_{2} \mathrm{O}_{2}$ stimulated MC-3T3-E1 cells with the cell permeant oxidation-sensitive dye DCF-DA. The present study indicates PI pretreatment effectively inhibited the RANKL and $\mathrm{H}_{2} \mathrm{O}_{2}$ induced ROS production both in OCs and OBs (Fig. 6).

\section{Discussion}

The maintenance of bone mass is severely affected in osteoporotic patients and other bone disorders and can have multiple etiologies. OCs differentiation (osteoclastogenesis) is frequently caused by excessive RANKL signaling which has been a valuable target for the treatment of pathological bone loss [19]. The present study of PI effects on osteoclastogenesis has revealed novel mechanisms of this natural plant for a potentially therapeutic application in treating bone loss disease. Previously, it has been reported that PI has anti-inflammatory properties and exerts beneficial effects in the cerebral [13]. Moreover, document studies have demonstrated picrasidine $\mathrm{N}$ and picrasidine $\mathrm{G}$ target multiple signaling pathways, such as: PARP-1and EGFR/STAT3 signaling pathway $[14,15]$. Here we have demonstrated for the first time that PI has effects on bone metabolism. Our results showed that PI not only inhibited multiple down stream signaling pathways of RANKL, which including MAPKs/cFos/NFATc1 and NF- $\kappa$ B, but also effectively decreased ROS in OBs for signal crosstalking of regulating osteoclastogenesis (Fig. 7).

Binding of RANKL to RANK is an essential initial step in the OCs development. It causes recruitment of TRAF 6 and activation of downstream critical signaling such as MAPKs and NF$\kappa B$ [25]. Moreover, activated NF- $\kappa B$ and MAPKs can translocate to the nucleus and regulate various transcription factors, including c-Fos and NFATc1 [26]. Therefore, to determine whether PI targeted c-Fos/NFATc1 by inhibiting MAPKs and NF- $\kappa B$ signaling cascades, we primarily examined the effects of PI treatment on the expression of c-Fos/NFATc1 during RANKL-induced osteoclastogenesis. Our study demonstrates the reduced c-Fos expression in PI treated OC precursor cells, which suggested cause by the impaired activation of NFATc1 responsible for the inhibition of RANKL-induced OCs formation. Moreover, MAPKs family members (p38, ERK and JNK) are proline-directed serine/threonine kinases that play important roles in OCs growth, differentiation, and apoptosis [27]. In that, ERK signaling is involved in the cell proliferation and differentiation, and the expression of p-ERK can induce the OCs proliferation and differentiation [28]. JNK signaling and p38 signaling play signaling a key role in the process of OCs formation, both of them can regulate downstream master transcription factors c-Fos/NFATc1 which can promote OCs differentiation [29]. By using the specific inhibitor of the p38, ERK and JNK, the results of our study demonstrated that PI inhibits RANKL-mediated OCs differentiation by inhibiting activation of the MAPKs. Furthermore, the classic NF- $\kappa B$ signaling pathway involves the activation of the inhibitor of $\kappa \mathrm{B}(\mathrm{I} \kappa \mathrm{B})$ kinase (IKK) complex that then phosphorylates I $\kappa \mathrm{Bs}$, targeting them for ubiquitindependent degradation [25]. Our results showed that PI suppressed the phosphorylation of p-p65, leading to reduced cytoplasmic degradation of I $\kappa B$ and the prevention of NF- $\kappa B^{\prime} s$ DNA-binding activity. These results demonstrated that the one of molecular mechanisms involved in PI inhibitory effects on osteoclastogenesis was initially via suppressing MAPKs and NF- $\kappa$ B siganling pathways then down-regulating the c-Fos/NFATc1.

Alternatively, OCs activity and function caused by various signaling pathways cross talking, previous study has demonstrated that ROS are involved in OCs activation and 


\section{Cellular Physiology Cell Physiol Biochem 2017;43:1425-1435 and Biochemistry \begin{tabular}{c|c|c|c|} 
DOI: 10.1159/000481874 & O 2017 The Author(s). Published by S. Karger AG, Basel \\
wubw.karger.com/cpb
\end{tabular} \\ Kong et al.: Agent from Picrasma Quassioides Suppress Osteoclastogenesis}

Fig. 7. PI inhibit osteoclastogenesis through multiple signaling pathways. PI treatment decreased the ROS generation on OB lineage cells. In OC precursors and OCs, PI repressed multiple pathways downstream of RANKL signaling, including $\mathrm{I} \kappa \mathrm{B} / \mathrm{NF}-\kappa \mathrm{B}, \mathrm{MAPKs}$, and c-Fos/NFATc1.

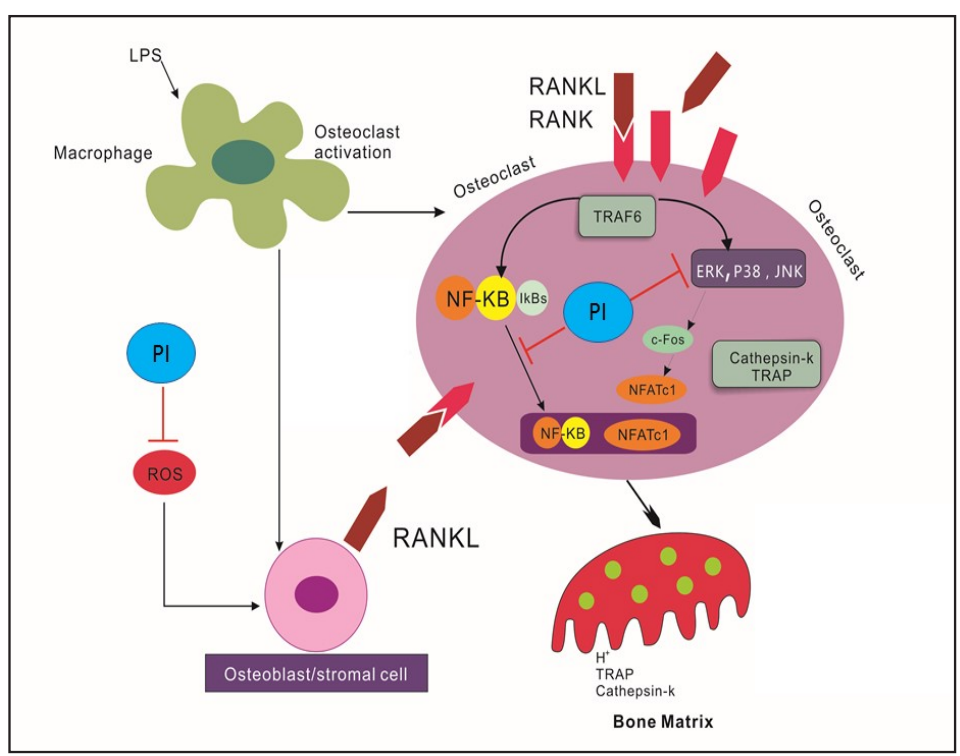

bone loss stimulated by increased expression of RANKL from stromal/OBs [30]. Moreover, antioxidant treatments have been proved to be effective to rescue the bone loss induced by oxidative stress by decreasing the NF- $\kappa \mathrm{B}$ activation in OBs and RANKL expression in OBs [31]. Owing to the bioactivity property of agents from picrasma quassioides, and in order to clarify whether the downregulation effects of PI on osteoclastogenesis cross-talk with ROS signalling. We have further evaluated the effects of PI on antioxidant system in RANKL stimulated RAW 264.7 and $\mathrm{H}_{2} \mathrm{O}_{2}$ stimulated OBs. We found that PI significantly decreased ROS production.

In summary, the present study indicates that PI attenuating osteoclastogenesis, possibly via inhibit the activation of MAPKs and NF- $\kappa B$ siganling pathways in BMMs and paralleled with attenuates ROS signalling pathway in OBs. Future research is needed to further explore the effect of PI on osteolysis in vivo, meanwhile, the pathways investigated in the current study should also be further confirmed. Our study highlights the bioactivity effects of agent from natural plant picrasma quassioides on bone metabolism, providing a new information of the PI on regulating osteoclastogenesis and identifying a potential natural compound for the treatment of osteoporosis.

\section{Acknowledgements}

This study was supported by a grant from China Postdoctoral Science Foundation, PR.China (2016M592825), grant from Shaanxi State Ministry of Health, PR.China (2016D044)

\section{Disclosure Statement}

The authors declare that they have no competing interests. All data and materials were included in the manuscript.

\section{References}

1 Segovia-Silvestre T, Neutzsky-Wulff AV, Sorensen MG, Christiansen C, Bollerslev J, Karsdal MA, Henriksen $\mathrm{K}$ : Advances in osteoclast biology resulting from the study of osteopetrotic mutations. Hum Genet 2009;124:561-577.

2 Chen F, Ni B, Yang YO, Ye T, Chen A: Knockout of TRPV6 causes osteopenia in mice by increasing osteoclastic differentiation and activity. Cell Physiol Biochem 2014;33:796-809. 


\section{Cellular Physiology Cell Physiol Biochem 2017;43:1425-1435

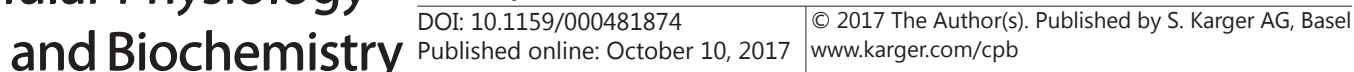

Kong et al.: Agent from Picrasma Quassioides Suppress Osteoclastogenesis

3 Boyle WJ, Simonet WS, Lacey DL: Osteoclast differentiation and activation. Nature 2003;423:337-342.

- 4 Lee SK, Park KK, Kim HJ, Kim KR, Kang EJ, Kim YL, Yoon H, Kim YS, Chung WY: Platycodin D Blocks Breast Cancer-Induced Bone Destruction by Inhibiting Osteoclastogenesis and the Growth of Breast Cancer Cells. Cell Physiol Biochem 2015;36:1809-1820.

5 Suda T, Takahashi N, Udagawa N, Jimi E, Gillespie MT, Martin TJ: Modulation of osteoclast differentiation and function by the new members of the tumor necrosis factor receptor and ligand families. Endocr Rev 1999;20:345-357.

-6 Wang L, Li SJ, Sidhu A, Zhu L, Liang Y, Freedman RB, Wang CC: Reconstitution of human Ero1-Lalpha/ protein-disulfide isomerase oxidative folding pathway in vitro. Position-dependent differences in role between the a and a' domains of protein-disulfide isomerase. J Biol Chem 2009;284:199-206.

-7 Hotokezaka H, Sakai E, Kanaoka K, Saito K, Matsuo K, Kitaura H, Yoshida N, Nakayama K: U0126 and PD98059, specific inhibitors of MEK, accelerate differentiation of RAW264.7 cells into osteoclast-like cells. J Biol Chem 2002;277:47366-47372.

-8 Matsuo K, Galson DL, Zhao C, Peng L, Laplace C, Wang KZ, Bachler MA, Amano H, Aburatani H, Ishikawa $\mathrm{H}$, Wagner EF: Nuclear factor of activated T-cells (NFAT) rescues osteoclastogenesis in precursors lacking c-Fos. J Biol Chem 2004;279:26475-26480.

9 Guo Y, Xu X, Li Q, Li Z, Du F: Anti-inflammation effects of picroside 2 in cerebral ischemic injury rats. Behav Brain Funct 2010;6:43.

10 Satoh T, McKercher SR, Lipton SA: Reprint of: Nrf2/ARE-mediated antioxidant actions of pro-electrophilic drugs. Free Radic Biol Med 2014;66:45-57.

11 Ayoub NA, Hussein SA, Hashim AN, Hegazi NM, Linscheid M, Harms M, Wende K, Lindequist U, Nawwar MA: Bone mineralization enhancing activity of a methoxyellagic acid glucoside from a Feijoa sellowiana leaf extract. Pharmazie 2009;64:137-141.

12 Zhao S, Kanno Y, Li W, Sasaki T, Zhang X, Wang J, Cheng M, Koike K, Nemoto K, Li H: Identification of Picrasidine C as a Subtype-Selective PPARalpha Agonist. J Nat Prod 2016;79:3127-3133.

13 Sasaki T, Li W, Ohmoto T, Koike K: Evaluation of canthinone alkaloids as cerebral protective agents. Bioorg Med Chem Lett 2016;26:4992-4995.

14 Zhao S, Kanno Y, Li W, Wakatabi H, Sasaki T, Koike K, Nemoto K, Li H: Picrasidine N Is a Subtype-Selective PPARbeta/delta Agonist. J Nat Prod 2016;79:879-885.

15 Yamashita N, Kondo M, Zhao S, Li W, Koike K, Nemoto K, Kanno Y: Picrasidine G decreases viability of MDA-MB 468 EGFR-overexpressing triple-negative breast cancer cells through inhibition of EGFR/STAT3 signaling pathway. Bioorg Med Chem Lett 2017;27:2608-2612.

16 Kong L, Yang C, Yu L, Smith W, Zhu S, Zhu J, Zhu Q: Pyrroloquinoline quinine inhibits RANKL-mediated expression of NFATc1 in part via suppression of c-Fos in mouse bone marrow cells and inhibits wear particle-induced osteolysis in mice. PLoS One 2013;8:e61013.

-17 Yang X, Gao W, Wang B, Wang X, Guo H, Xiao Y, Kong L, Hao D: Picroside II Inhibits RANKL-Mediated Osteoclastogenesis by Attenuating the NF-kappaB and MAPKs Signaling Pathway In vitro and Prevents Bone Loss in Lipopolysaccharide Treatment Mice. J Cell Biochem 2017;10.1002/jcb.26105

18 Kong L, Ma R, Yang X, Zhu Z, Guo H, He B, Wang B, Hao D: Psoralidin suppresses osteoclastogenesis in BMMs and attenuates LPS-mediated osteolysis by inhibiting inflammatory cytokines. Int Immunopharmacol 2017;51:31-39.

19 Guan H, Zhao L, Cao H, Chen A, Xiao J: Epoxyeicosanoids suppress osteoclastogenesis and prevent ovariectomy-induced bone loss. FASEB J 2015;29:1092-1101.

-20 Shrivastava S, Kulkarni P, Thummuri D, Jeengar MK, Naidu VG, Alvala M, Redddy GB, Ramakrishna S: Piperlongumine, an alkaloid causes inhibition of PI3 K/Akt/mTOR signaling axis to induce caspasedependent apoptosis in human triple-negative breast cancer cells. Apoptosis 2014;19:1148-1164.

21 Baek JM, Kim JY, Jung Y, Moon SH, Choi MK, Kim SH, Lee MS, Kim I, Oh J: Mollugin from Rubea cordifolia suppresses receptor activator of nuclear factor-kappaB ligand-induced osteoclastogenesis and bone resorbing activity in vitro and prevents lipopolysaccharide-induced bone loss in vivo. Phytomedicine 2015;22:27-35.

22 Tseng FJ, Chia WT, Wang CH, Shyu JF, Gou GH, Shui HA, Sytwu HK, Pan RY, Weng CF: Carbon Monoxide Inhibits Receptor Activator of NF-kappaB (RANKL)-Induced Osteoclastogenesis. Cell Physiol Biochem 2015;36:1250-1258. 


\section{Cellular Physiology Cell Physiol Biochem 2017;43:1425-1435 \begin{tabular}{l|l|l} 
DOI: 10.1159/000481874 & $\begin{array}{l}\text { O 2017 The Author(s). Published by S. Karger AG, Basel } \\
\text { www.karger.com/cpb }\end{array}$ \\
\hline
\end{tabular}}

Kong et al.: Agent from Picrasma Quassioides Suppress Osteoclastogenesis

23 Guo C, Hou GQ Li XD, Xia X, Liu DX, Huang DY, Du SX: Quercetin triggers apoptosis of lipopolysaccharide (LPS)-induced osteoclasts and inhibits bone resorption in RAW264.7 cells. Cell Physiol Biochem 2012;30:123-136.

24 Lin S, Qiu M, Chen J: IL-4 Modulates Macrophage Polarization in Ankylosing Spondylitis. Cell Physiol Biochem 2015;35:2213-2222.

25 Vaira S, Alhawagri M, Anwisye I, Kitaura H, Faccio R, Novack DV: RelA/p65 promotes osteoclast differentiation by blocking a RANKL-induced apoptotic JNK pathway in mice. J Clin Invest 2008;118:20882097.

26 Takayanagi H: Osteoimmunology: shared mechanisms and crosstalk between the immune and bone systems. Nat Rev Immunol 2007;7:292-304.

27 Heo JS, Han HJ: PKC and MAPKs pathways mediate EGF-induced stimulation of 2-deoxyglucose uptake in mouse embryonic stem cells. Cell Physiol Biochem 2006;17:145-158.

28 Thoresen GH, Guren TK, Christoffersen T: Role of ERK, p38 and PI3-kinase in EGF receptor-mediated mitogenic signalling in cultured rat hepatocytes: requirement for sustained ERK activation. Cell Physiol Biochem 2003;13:229-238.

29 Agron M, Brekhman V, Morgenstern D, Lotan T: Regulation of AP-1 by MAPK Signaling in Metal-Stressed Sea Anemone. Cell Physiol Biochem 2017;42:952-964.

-30 Lee NK, Choi YG, Baik JY, Han SY, Jeong DW, Bae YS, Kim N, Lee SY: A crucial role for reactive oxygen species in RANKL-induced osteoclast differentiation. Blood 2005;106:852-859.

-31 Monjo M, Rubert M, Ellingsen JE, Lyngstadaas SP: Rosuvastatin promotes osteoblast differentiation and regulates SLC01A1 transporter gene expression in MC3T3-E1 cells. Cell Physiol Biochem 2010;26:647-656. 